\title{
BI Lyncis: A hydrogen-deficient binary consisting of two low-mass giants of spectral types early-B and $G^{\star}$
}

\author{
C. S. Jeffery and R. Aznar Cuadrado
}

Armagh Observatory, College Hill, Armagh BT61 9DG, Northern Ireland

Received 21 June 2001 / Accepted 4 September 2001

\begin{abstract}
BI Lyn has previously been misclassified as an evolved binary system containing either a hot subdwarf or white dwarf and a thick accretion disk. New intermediate dispersion spectra are presented which demonstrate clearly that the hot component is a luminous low-mass helium star and the cool companion is a rapidly rotating G-type giant. Techniques of spectrum synthesis have been used to establish the dimensions of both components. Although the orbital period of the system remains unknown, other phenomena are entirely consistent with these observed dimensions. A $\sim 0.34 \mathrm{~d}$ periodic photometric variation could be explained by pulsations in the B-type primary and, by analogy with other $\mathrm{H}$-deficient binaries, it is suggested that the variable absorption in $\mathrm{H} \alpha$ may be due to a gas stream flowing through the inner Lagrangian point.
\end{abstract}

Key words. stars: fundamental parameters - stars: binaries - stars: chemically peculiar - stars: individual: BI Lyn

\section{Introduction}

BILyn (=PG0900+400) has been identified as a composite binary containing a hot subdwarf primary with effective temperature $T_{\text {eff }} \sim 31000 \mathrm{~K}$ and a cool mainsequence star of spectral type $\sim$ K3 (Ferguson et al. 1984). As such, it was proposed to be the potential progenitor of a cataclysmic variable (CV) system. The subsequent passage from being a suspected CV progenitor to becoming a "nova-like" CV (Downes et al. 1997; Ritter \& Kolb 1998) appears to have come from the juxtaposition of a small-amplitude light variation (Lipunova \& Shugarov 1991) with a suggested thick accretion disk (Ferguson et al. 1984) by compilers of the variable star lists (Kazarovets et al. 1993). There is, however, no evidence for any long-term variability, although there may be a 0.338 day photometric variation (Lipunova \& Shugarov 1990; Lipunova \& Shugarov 1991; Kuczawska et al. 1993). It was only following a more detailed spectroscopic study that firm evidence of variability, manifest in the behaviour of the blue-shifted $\mathrm{H} \alpha$ absorption component of a broader emission line, was obtained (Wade \& Potter 1995). Deconvolution of the

Send offprint requests to: C. S. Jeffery,

e-mail: csj@star.arm.ac.uk

* Based on observations obtained with the Isaac Newton and William Herschel Telescopes, and on INES data from the IUE satellite. composite spectra confirmed the spectral types of the hot and cool components (Orosz et al. 1997).

BI Lyn stands out amongst the composite stars of Ferguson et al. (1984) for having a distinctly red flux distribution, where all other composites have blue or flat spectra. Orosz et al. (1997) refer to PG0900+400 specifically for several reasons. It is the only member of their own sample to show $\mathrm{H} \alpha$ emission. It appears to have an unusually large rotation velocity $\left(v \sin i>130 \mathrm{~km} \mathrm{~s}^{-1}\right)$. In contrast to other stars in their sample, which show the He I $\mathrm{D}_{3}$ absorption near $5876 \AA$ with equivalent widths between 0.5 and $1.0 \AA$, the $\mathrm{D}_{3}$ line in $\mathrm{PG} 0900+400$ has an equivalent width of $2.0 \AA$ or more. Low-resolution spectroscopy (Kuczawska et al. 1993; Liu \& Hu 2000) also points to numerous prominent HeI lines which are stronger than neighbouring Balmer lines.

Taken overall, the literature on BI Lyn suggests a hot helium-rich subdwarf with a K-type main-sequence companion or other cool source. Broad absorption lines, variable $\mathrm{H} \alpha$ emission and a $0.34 \mathrm{~d}$ light variation suggest that the latter could include a thick accretion disk. In evolutionary terms it would lie between a common-envelope phase (red giant plus main sequence star) and a cataclysmic variable (white dwarf plus main sequence star). Being helium rich, it could represent a very important phase of binary evolution.

The star caught our attention during a new intermediate-dispersion survey of composite spectrum subdwarfs. Fortunately, we had overlooked the SIMBAD 
Table 1. Spectroscopic observations of BI Lyn.

\begin{tabular}{|c|c|c|c|c|}
\hline Image & $\begin{array}{r}\text { Date: JD } \\
-2450000\end{array}$ & $\begin{array}{c}\text { Wavelength } \\
\AA\end{array}$ & $\begin{array}{c}\text { Res. } \\
\text { exp } \\
\text { s }\end{array}$ \\
\hline INT+IDS \\
\hline 155679 & 1264.427 & $3820-4686$ & 2500 & 300 \\
155725 & 1264.547 & $6070-6930$ & 3900 & 300 \\
155928 & 1264.553 & $7975-8813$ & 5100 & 300 \\
\hline WHT+ISIS & 1265.603 & $3821-4687$ & 2500 & 300 \\
\hline 337312 & 1676.371 & $4246-4653$ & 5600 & 100 \\
337313 & 1676.374 & $4246-4653$ & 5600 & 200 \\
337318 & 1676.383 & $3898-4304$ & 5100 & 200 \\
337319 & 1676.385 & $3898-4304$ & 5100 & 200 \\
337414 & 1677.358 & $6364-6769$ & 8300 & 61 \\
337415 & 1677.359 & $6364-6769$ & 8300 & 200 \\
337416 & 1677.360 & $4597-5004$ & 6000 & 200 \\
337417 & 1677.363 & $6364-6769$ & 8300 & 200 \\
337418 & 1677.363 & $4597-5004$ & 6000 & 200 \\
337419 & 1677.367 & $4246-4653$ & 5600 & 200 \\
337420 & 1677.367 & $8408-8803$ & 11100 & 300 \\
\hline
\end{tabular}

designation "V* BI Lyn - Nova-like Star" and had retained the star in our sample. In our ignorance of the literature discussed above, an immediate examination of the new spectra suggested a quite different interpretation. This has been fully confirmed by subsequent observations and more detailed analysis.

\section{Observations}

\subsection{INT}

Intermediate dispersion spectroscopy of a sample of candidate subdwarf B stars with cool companions was carried out with the Isaac Newton telescope on 1999 March 26 and 27, using the $235 \mathrm{~mm}$ camera of the Intermediate Dispersion Spectrograph, R1200R and R1200B diffraction gratings and the TEK5 $(1124 \times 1124)$ CCD. Wavelength regions were chosen to determine the atmospheric parameters of the sdB stars (blue) and their cool companions (infrared calcium triplet). Red spectra (around $\mathrm{H} \alpha$ ) were obtained in an effort to obtain spectrophotometry of the cool companions (cf. Jeffery \& Pollacco 1998). All observations and data reduction followed standard procedures, including bias-subtraction, flat-fielding and wavelength calibration, and are described in full by Aznar Cuadrado (Aznar Cuadrado 2001). Details of the observations of BI Lyn are given in Table 1 and the spectra are shown in Fig. 1.

Our first reaction upon seeing the quick-look extraction of the blue spectrum of PG0900+400 was that we were looking at an extreme helium star. This was in
Table 2. Heliocentric radial velocity measurements of BI Lyn.

\begin{tabular}{|c|r|rr|rr|r|}
\hline Image & Date: JD & \multicolumn{2}{|c|}{ B star } & \multicolumn{2}{|c|}{ G star } & \\
& -2450000 & $v$ & \pm & $v$ & \pm & $\delta v$ \\
\hline$\sim 3900-4900 \AA$ \\
\hline 155679 & 1264.427 & 36.8 & 3.1 & 24.9 & 3.4 & 12.0 \\
155928 & 1265.603 & 37.8 & 4.1 & 21.4 & 2.1 & 16.4 \\
337312 & 1676.371 & 28.1 & 1.6 & 39.1 & 3.1 & -11.0 \\
337313 & 1676.374 & 17.7 & 3.4 & 39.0 & 3.1 & -21.3 \\
337318 & 1676.383 & 40.5 & 3.1 & 13.6 & 0.8 & 26.9 \\
337319 & 1676.385 & 40.3 & 2.5 & 15.4 & 1.4 & 24.9 \\
337416 & 1677.360 & 10.5 & 3.0 & 34.6 & 2.6 & -24.1 \\
337418 & 1677.363 & 19.6 & 3.4 & 34.4 & 2.1 & -14.8 \\
\hline$\sim$ CaT & \multicolumn{7}{|c|}{-1264.553} & - & - & 19.3 & 0.2 & - \\
\hline 155728 & 1267.367 & - & - & 27.3 & 0.5 & - \\
337420 & 1677.367 & \\
\hline Mean & & 28.9 & 26.9 & 1.1 \\
\pm & & 11.7 & 9.5 & 21.1 \\
\hline
\end{tabular}
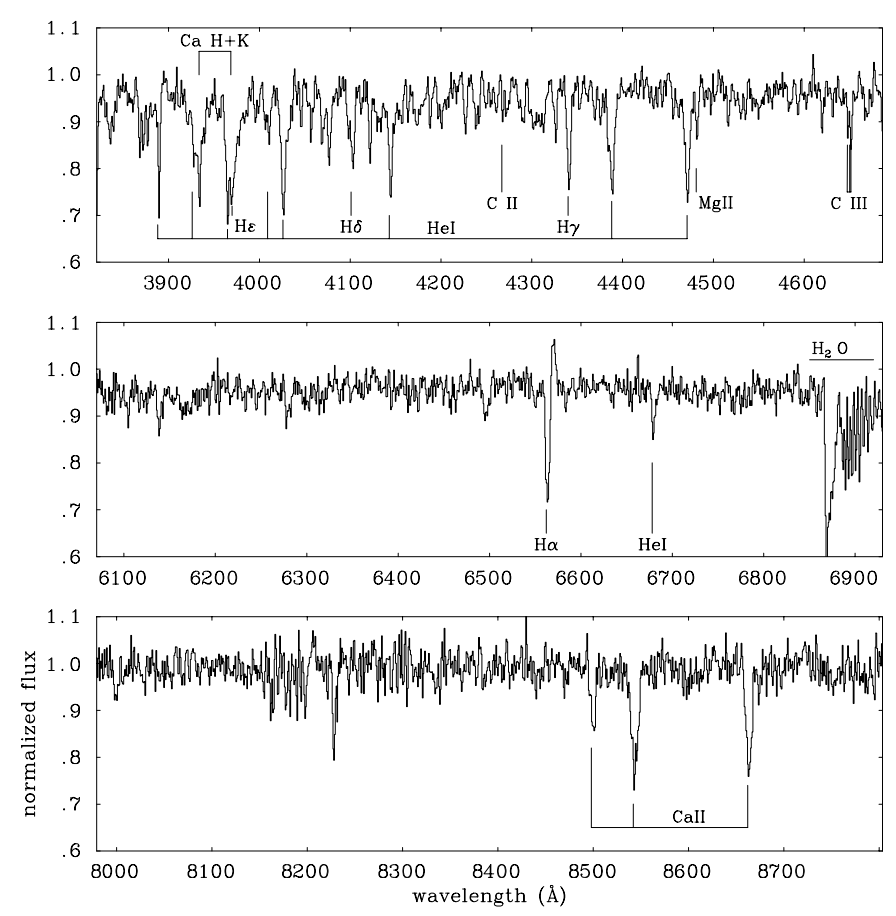

Fig. 1. INT intermediate-resolution spectra of BI Lyn, showing sharp He I lines, weaker Balmer lines, a veil of metal lines from a cool component (top: INT images 155679+155928), a P Cygni profile in $\mathrm{H} \alpha$ (middle: 155725 ) and the calcium infrared triplet (bottom: 155728). The spectra have been normalized to an estimated continuum. Some of the principal absorption lines are labelled.

ignorance of the history described in the introduction and on the expectation that we should see a typical subdwarf B spectrum with strong and broad Balmer lines superposed by a weak cool-star spectrum. In fact, the neutral 
helium spectrum stands out clearly while the Balmer lines are weak and narrow. Other features include C II $\lambda 4267 \AA$, Mg II $\lambda 4481 \AA$ and Ca H+K (Fig. 1). Meanwhile the rest of the spectrum is very rich in absorption lines. The authors were immediately reminded of the spectra of cool helium stars such as PV Tel and $v$ Sgr (Jeffery et al. 1987). Closer inspection disavowed this conclusion and raised several difficulties.

First of all we consider the evidence of the helium lines in the blue. There is no evidence for the Pickering series of He II, so the Balmer lines are due to hydrogen and the effective temperature cannot exceed $35000 \mathrm{~K}$. The fact that He I $\lambda 4388 \AA$ is considerably stronger than $\mathrm{H} \gamma$ implies that the star is hydrogen deficient. The ratio of He I $\lambda 4471 \AA$ to Mg II $\lambda 4481 \AA$ requires that the temperature of the helium star must be higher than that of PV Tel $(15000 \mathrm{~K}$ : Walker \& Schönberner 1981).

The calcium infrared triplet is strong (Fig. 1), proof positive of a star with spectral type $\mathrm{G}$ or later, even given the peculiar properties of stellar spectra in the absence of hydrogen.

Having examined the literature and discovered that we were not the first to encounter these ambiguities, we concluded that we must be looking at a composite spectrum binary containing a helium star and, given previous work, a $\mathrm{G}$ or $\mathrm{K}$ star. The question that arises is whether we are looking at two giants (or supergiants), or at two dwarfs. The possibility that both components are giants is intriguing. $\mathrm{H} \alpha$ emission (Fig. 1) has in fact been seen elsewhere in hydrogen-deficient giants such as $v$ Sgr (Seydel 1929).

A preliminary analysis based on these data (Aznar Cuadrado et al. 12th EADN Predoctoral School. Selected topics on binary stars. Observation and physical processes. La Laguna, Spain. September 6-17 1999, unpublished) failed to resolve the surface gravities of both cool and hot components in a manner consistent with the relative angular radii determined from spectrophotometry and with sensible values of the masses for both stars. A likely reason was that the cool-star spectrum contaminates the hotstar spectrum so severely that the lines are simply too heavily blended at this spectral resolution. Consequently, spectral fitting by least-squares minimisation was poorly constrained. It was therefore necessary to obtain higher resolution spectra, and to combine these with previous ultraviolet and visual spectrophotometry.

\subsection{WHT observations}

Further intermediate dispersion spectroscopy of BI Lyn was carried out with the William Herschel telescope on 2000 May 11 and 12, using ISIS, R1200R and R1200B diffraction gratings and the TEK2 (red arm) and TEK4 (blue arm) $(1124 \times 1124)$ CCDs. With approximately double the spectral resolution, similar wavelength regions were chosen as before. Details are given in Table 1. All observations and data reduction followed standard procedures, including bias-subtraction, flat-fielding and wavelength calibration, are again described in full by Aznar Cuadrado (Aznar Cuadrado 2001).

Where possible, spectra were obtained in pairs to avoid the risk of a cosmic ray hit on an important line. These multiple spectra were coadded. In addition, data from three contiguous wavelength regions in the blue were combined to form a single spectrum $(\lambda$ 3900-5000 $\AA)$ for use in the subsequent analysis. Each region was normalized by fitting a low-order polynomial to regions of pseudo-continuum. Overlapping wavelength regions were inspected to ensure that the overlap was good before merging the individual sections. If necessary, the pseudo-continuum was adjusted to ensure this condition was fulfilled. The result was three spectra for analysis with wavelength ranges $3900-5000 \AA$, 6360-6760 $\AA$ and 8400-8800 $\AA$. Unfortunately the $8400-8800 \AA$ was too noisy and contaminated by flat-field fringes to be useful for spectral analysis, although it could be used for radial velocity measurements.

\subsection{Spectrophotometry}

BI Lyn was observed within two IUE programmes in 1995. The low-resolution image numbers are LWP30587, SWP54558, SWP54559, SWP54560, SWP56235, of which SWP54559 and SWP54560 were made with the small aperture and the remainder with the large aperture. These images have been recovered from the IUE Final Archive as Newly Extracted Spectra. The short-wavelength IUE spectrum is typical of a hot subdwarf, showing very strong He II, C III, IV, N V, and Si IV absorption lines.

Broad-band colours have been reported by Ferguson et al. (1984) and by Lipunova \& Shugarov (1991), from which we have adopted $V=12.85, B-V=0.2, U-B=$ $-0.96, V-R=0.3$ and $V-I=0.58$.

By combining these data, a single description of the flux distribution between 1100 and $9000 \AA$ has been derived (Fig. 2). For the time being, we have chosen to discount the possibility of variability in the overall flux distribution. If present, it does not exceed 0.2 mag (Lipunova \& Shugarov 1990) in $V$.

\subsection{Radial velocities}

Radial velocities were measured by cross-correlation. The cross-correlation templates were taken to be the best-fit model spectra for each stellar component as described in the next section. Since a velocity needs to be known a priori to perform this analysis, a two-step iteration for velocity was effected. Strong lines common to both stars (e.g. Balmer lines) were excluded from the cross-correlation. The heliocentric velocities measured from each observation are given in Table 2. Errors on individual velocities are formal errors from fitting a Gaussian to the ccf peak. The actual errors are probably much larger, but difficult to determine quantitatively; the typical ccf width is 


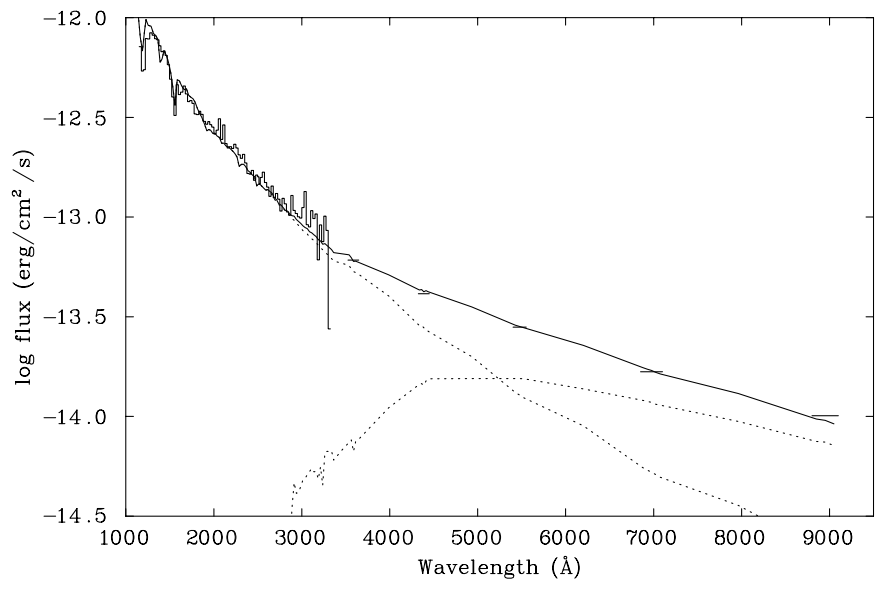

Fig. 2. Ultraviolet and visual spectrophotometry of BI Lyn (histogram) together with the best fitting theoretical flux distribution (polyline and horizontal bars). The latter represents the sum of two model atmospheres (dashed lines) with effective temperature and angular radii $T_{\text {eff } 1}=28600 \mathrm{~K}, \theta_{1}=$ $0.55 \times 10^{-11} \mathrm{rad}, T_{\text {eff } 2}=5840 \mathrm{~K}$ and $\theta_{2}=4.09 \times 10^{-11} \mathrm{rad}$. Interstellar reddening is negligible.

200-300 $\mathrm{km} \mathrm{s}^{-1}$. The standard deviation about the mean $\left(\sim 10 \mathrm{~km} \mathrm{~s}^{-1}\right)$ may be a better indication.

Wavelength calibration and other systematic errors may be responsible for anomalous measurements from spectra around $\mathrm{H} \alpha$ and one blue spectrum (WHT image 337419). These are not shown in Table 2.

The average velocities for both components, 29 and $27 \mathrm{~km} \mathrm{~s}^{-1}$, respectively, are consistent with previous velocity measurements (Orosz et al. 1997).

\section{Analysis}

The available spectra of BI Lyn suggest that it comprises a hot helium-rich source and a cooler source since we see absorption lines due to plasma in two quite different ionization states. In constructing a model to fit these spectra, a number of assumptions - or approximations - influence our conclusions. Many of these are necessary because both the number of free parameters and the limited number of constraints preclude an exhaustive search of solution space.

The fundamental assumption is that both cool and hot absorption sources are primarily stellar. This seems to hold well for the hot source. This remains a good working hypothesis even though high rotational broadening in the cool source spectrum and the presence of $\mathrm{H} \alpha$ emission makes a purely stellar identification less secure.

For two stellar sources, the principal free parameters which govern the measured spectrum are as follows (subscripts refer to the hot and cool source respectively):

- $T_{\text {eff } 1}, T_{\text {eff } 2}$ : effective temperature,

- $E_{B-V}$ : interstellar extinction,

- $\log g_{1}, \log g_{2}:$ surface gravity,

$-\theta_{1}, \theta_{2}$ : angular diameter providing the relative radii $R_{2} / R_{1}$
- $v \sin i_{1}, v \sin i_{2}$ : rotational velocity,

$-v_{1}, v_{2}$ : radial velocity,

- $n_{\mathrm{He} 1}, n_{\mathrm{He} 2}$ : helium abundance,

$-\left[\beta_{\mathrm{Fe}}\right]_{1},\left[\beta_{\mathrm{Fe}}\right]_{2}:$ metallicity $^{1}$,

$-v_{\mathrm{t} 1}, v_{\mathrm{t} 2}$ : microturbulent velocity.

Ideally, stellar composition entails many more free parameters than metallicity and helium abundance alone, but requires high-resolution spectroscopy to measure, as does the microturbulent velocity. Helium abundance cannot be measured directly for cool sources. Tests showed that $\left[\beta_{\mathrm{Fe}}\right]_{2}$ cannot be uniquely determined from the given data. Similarly, given the magnitude of errors in $T_{\text {eff } 1}$, it is not practical to measure $\left[\beta_{\mathrm{Fe}}\right]_{1}$ in detail.

Thus the second group of assumptions are as follows. The abundances of all elements other than hydrogen and helium are in proportion to their cosmic abundances, with $\left[\beta_{\mathrm{Fe}}\right]_{1}=\left[\beta_{\mathrm{Fe}}\right]_{2}=0$. The helium abundance of the cool star is normal: $n_{\mathrm{He} 2}=n_{\mathrm{He} \odot}$. The adopted microturbulent velocities are typical for early-type stars $v_{\mathrm{t} 1}=5 \mathrm{kms}^{-1}$ and main-sequence late-type stars $v_{\mathrm{t} 2}=2 \mathrm{kms}^{-1}$. The latter assumption is very important as it affects both the metallicity $\left[\beta_{\mathrm{Fe}}\right]_{2}$ (see above) and the derived radius ratio $R_{2} / R_{1}$. We have adopted $v_{\mathrm{t} 2}=2 \mathrm{~km} \mathrm{~s}^{-1}$ in order that the latter quantity as derived from spectral fitting be as consistent as possible with $\theta_{2} / \theta_{1}$ derived from spectrophotometry where we have also used cool star models computed with $v_{\mathrm{t} 2}=2 \mathrm{~km} \mathrm{~s}^{-1}$. Secondary effects on $T_{\mathrm{eff} 2}$ and $\log g_{2}$ are not significant here.

The third level of assumptions concerns the physics. The stars are assumed to be spherically symmetric and not to vary significantly over time. The approximations of plane-parallel geometry, local thermodynamic,

1 The measurement of chemical abundance from a spectral line normally provides the number fraction $(n)$ relative to the continuous opacity source which, in most stars, is hydrogen. The metallicity of a star may then be given as the logarithm of the ratio of the iron-to-hydrogen fraction $\left(n_{\mathrm{Fe}} / n_{\mathrm{H}}\right)$ relative to that in the Sun: $[\mathrm{Fe} / \mathrm{H}] \equiv \log \left(\left(n_{\mathrm{Fe}} / n_{\mathrm{H}}\right) /\left(n_{\mathrm{Fe}} / n_{\mathrm{H}}\right)_{\odot}\right)$. Thus $[\mathrm{Fe} / \mathrm{H}]=-0.3$ implies one half of the solar metallicity, assuming that the relative abundances of species heavier than helium follow some cosmic norm. These conventions break down for hydrogen-deficient stars, where nucleosynthesis has replaced hydrogen with helium and other heavy elements, and continuous opacity is provided by other species. In some cases, it makes sense to cite fractional abundance by number relative to the total number of ions in the plasma (e.g. $n_{\mathrm{Fe}}$ ). Where this is done for normal stars, the logarithm is taken and an arbitrary constant $c$ added to make the hydrogen abundance $\epsilon_{\mathrm{H}} \equiv \log n_{\mathrm{H}}+c=12.00$. This number can be used in stars where hydrogen is replaced by helium, but the constant must be modified to reflect the change in mass fractions of $\mathrm{H}$, He and possibly other species. Mass fractions $(\beta)$ provide a more convenient measure because they are conserved when other species transmute, but they are less often used in the literature. For this paper we adopt the convention $\left[\beta_{\mathrm{Fe}}\right]=\log \left(\beta_{\mathrm{Fe}} / \beta_{\mathrm{Fe} \odot}\right)$ to refer to the mass fraction of iron relative to that in the Sun. Thus $\left[\beta_{\mathrm{Fe}}\right]=0.0$ correctly implies a solar metallicity, regardless of the abundances of transmuted elements. For normal stars, $\left[\beta_{\mathrm{Fe}}\right] \equiv[\mathrm{Fe} / \mathrm{H}]$. 
radiative and hydrostatic equilibrium have been assumed valid for modelling their atmospheres (rapid rotation and $\mathrm{H} \alpha$ emission may compromise these).

However, with these assumptions and approximations, it becomes possible, in principle, to solve for the remaining 12 (!) free parameters by modelling the overall flux distribution and the intermediate-resolution spectra of BI Lyn.

\subsection{Spectral fitting: TFIT and SFIT}

The methods for fitting flux distributions and highresolution spectra by least-squares minimization within grids of models have been described in detail elsewhere (Jeffery et al. 2001). The primary codes used are BINFIT and SFIT.

BINFIT is an extension of the single star code TFIT used to model the flux distribution of a binary system containing a hot and a cool star. Fitting of each component is done within a one-dimensional grid, normally $T_{\text {eff }}$, convolved with an extinction curve $a_{\lambda}\left(E_{B-V}\right)$ and angular radius $\theta$. The flux distributions used in the model grids are of low resolution and taken directly from the output of LTE model atmosphere codes STERNE (Jeffery et al. 2001) or ATLAS (Kurucz 1979).

SFIT is used to model intermediate and high-resolution stellar spectra by interpolation in three-dimensional model grids, normally $T_{\text {eff }}, \log g$ and an abundance parameter, e.g. $n_{\mathrm{He}}$ or $\left[\beta_{\mathrm{Fe}}\right]$. Versions exist for both single and binary stars, and appropriate allowance is made for velocity shifts, rotational broadening, and instrumental broadening. Given the relative radii of stars in a binary, the spectra are added correctly at the absolute flux level and then normalized to the true total continuum. Provision is made to renormalize the observed spectrum to the true continuum in order to optimize the fit. SFIT may be used to measure any or all of $T_{\text {eff }}, \log g$, abundance, $v \sin i$, and $R_{2} / R_{1}$. In practise, only two or three variables should be solved for simultaneously, although $R_{2} / R_{1}$ should always be a free parameter. Consequently, the use of SFIT is iterative, particularly for binaries.

The radial velocity shifts $v$ may be found by crosscorrelating the observed spectrum with individual components of a synthetic spectrum which is a sufficiently good approximation to the final solution. Providing the hot and cool star spectra are sufficiently different, cross-correlation will automatically identify the individual components in the observed spectrum, although it is important to exclude strong features present in both spectra (e.g. Balmer lines).

\subsection{The model grids}

The model atmospheres and flux distributions used to analyse the hot star are computed with the planeparallel LTE code STERNE, which is adapted for dealing with hydrogen-deficient stellar atmospheres. The highresolution spectra are calculated with the LTE code
Table 3. Best solution for BI Lyn. See text for explanation of model fit parameters.

\begin{tabular}{|l|cc|cc|l|}
\hline Star & 1 & \pm & 2 & \pm & \\
\hline \multicolumn{5}{|l|}{ Spectrophotometry } \\
\hline$E_{B-V}$ & 0.00 & 0.02 & & & \\
$T_{\text {eff }}$ & 28.6 & 1.0 & 5.84 & 0.96 & $\mathrm{kK}$ \\
$\theta$ & 0.55 & 0.01 & 4.09 & 0.10 & $10^{-11} \mathrm{rad}$ \\
$v_{\mathrm{t}}$ & 5 & $a$ & 2 & $a$ & $\mathrm{~km} \mathrm{~s}^{-1}$ \\
$R / R_{1}$ & 1 & & 7.44 & 0.03 & \\
\hline Spectroscopy & & \multicolumn{3}{l|}{} \\
\hline$\left(T_{\text {eff }}\right.$ & 30.1 & 0.01 & & & $\mathrm{kK})^{b}$ \\
$T_{\text {eff }}$ & 28.6 & $a$ & 5.84 & $a$ & $\mathrm{kK}^{\prime}$ \\
$\log g$ & 3.6 & 0.1 & 3.2 & 0.3 & $\left(\mathrm{cgs}^{2}\right.$ \\
$n_{\mathrm{He}}$ & 0.95 & 0.01 & 0.1 & $a$ & \\
{$\left[\beta_{\mathrm{Fe}}\right]$} & 0.0 & $a$ & 0.0 & $a$ & \\
$v \sin i$ & 0 & $a$ & 120 & 20 & $\mathrm{~km} \mathrm{~s}^{-1}$ \\
$v_{\mathrm{t}}$ & 5 & $a$ & 2 & $a$ & $\mathrm{~km} \mathrm{~s}^{-1}$ \\
$R / R_{1}$ & 1 & & 4.9 & 0.5 & \\
\hline
\end{tabular}

$\begin{array}{ll}{ }^{a} & \text { Assumed value. } \\ { }^{b} & \text { Free solution not used. }\end{array}$

SPECTRUM. Both codes are described more fully by Jeffery et al. (2001).

Model atmospheres used in this investigation were calculated on a three-dimensional rectangular grid defined by $T_{\text {eff }}=10000(5000) 40000 \mathrm{~K}, \log g=1.0(0.5) 6.0$, and composition $\left[n_{\mathrm{H}}, n_{\mathrm{He}},\left[\beta_{\mathrm{Fe}}\right]\right]=[0.0,1.0,0.0]$, $[0.01,0.99,0.0],[0.05,0.95,0.0]$ and $[0.1,0.9,-1.0]$. The larger value of $n_{\mathrm{H}}$ or $n_{\mathrm{He}}$ is reduced to compensate for the trace elements. Coarse model grids for $n_{\mathrm{H}}=0.3,0.5$, $0.7,0.9,0.95,0.99$ and 1.0 , with corresponding $n_{\mathrm{He}}=$ $1-n_{\mathrm{H}}$ and $\left[\beta_{\mathrm{Fe}}\right]$, are also available, as are fine grids with $\delta T_{\text {eff }}=1000 \mathrm{~K}$ and $\delta \log g=0.1$ for selected areas of $\left(T_{\text {eff }}, \log g\right)$ space.

Synthetic spectra were calculated on wavelength in-

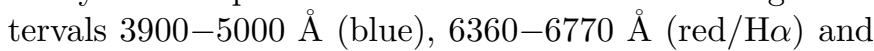
8400-8800 $\AA(\mathrm{CaT})$. Linelists were taken from the list of assessed data for hot stars LTE_LINES (Jeffery 1991). Microturbulent velocity $v_{\mathrm{t}}=5.0 \mathrm{~km} \mathrm{~s}^{-1}$ and solar abundances for all elements other than hydrogen and helium were assumed (see above).

Model atmospheres and flux distributions used to analyse the cool star were taken from the Kurucz' standard grid of ATLAS models (Kurucz 1993), for $T_{\text {eff }}=3500(500) 8000, \log g=2.0(0.5) 4.5, \quad\left[\beta_{\mathrm{Fe}}\right]=$ $-0.5,-0.3,0.0$ and $v_{\mathrm{t}}=2.0 \mathrm{~km} \mathrm{~s}^{-1}$.

High resolution spectra were calculated in the same spectral regions as for the hot star using Kurucz' code SYNTHE (Kurucz 1991; Jeffery et al. 1996). Grids with microturbulent velocities $v_{\mathrm{t}}=2,5$ and $10.0 \mathrm{~km} \mathrm{~s}^{-1}$ were computed. 
Table 4. Fundamental properties for stellar components of BI Lyn derived from Table 3 assuming two different values for the radius ratio $R_{2} / R_{1}$, the hot star mass $M_{1}$ and the cool star gravity $\log g_{2}$.

\begin{tabular}{|l|cc|cc|cc|}
\hline \multicolumn{7}{|l|}{ Assumed values } \\
\hline & $4.9^{a}$ & 0.5 & $7.4^{b}$ & 0.03 & $4.9^{a}$ & 0.5 \\
$R_{2} / R_{1}$ & 0.5 & 0.05 & 0.5 & 0.05 & 1.0 & 0.1 \\
$M_{1} / M_{\odot}{ }^{c}$ & 1.85 & 0.34 & 1.85 & 0.34 & 2.62 & 0.48 \\
\hline Derived values & 3.32 & 0.10 & 3.32 & 0.10 & 3.62 & 0.10 \\
\hline$R_{1} / R_{\odot}$ & 9.09 & 1.89 & 13.80 & 2.51 & 12.85 & 2.68 \\
$\log L_{1} / L_{\odot}$ & 1.93 & 0.11 & 2.29 & 0.10 & 2.23 & 0.11 \\
$R_{2} / R_{\odot}$ & 5.01 & 1.05 & 7.61 & 1.40 & 7.09 & 1.49 \\
$\log L_{2} / L_{\odot}$ & 4.8 & 1.8 & 11.0 & 3.9 & 9.6 & 3.5 \\
$d / \operatorname{kpc}$ & 1.0 & 0.4 & 2.2 & 0.8 & 1.9 & 0.7 \\
$M_{2} / M_{\odot}\left(\log g_{2}=3.2\right)$ &
\end{tabular}

${ }^{a} R_{2} / R_{1}$ from spectroscopy.

${ }^{b} R_{2} / R_{1}$ from spectrophotometry.

${ }^{c}$ Trial values.

\subsection{Spectrophotometry}

The method for measuring $T_{\text {eff }}$ and angular radii $\theta$ for both components in composite systems containing a hot subdwarf and a cool companion using IUE spectrophotometry and optical-IR broad-band photometry has been described elsewhere (Aznar Cuadrado \& Jeffery 2001). In fact PG0900+400 appeared in the sample analyzed, yielding $T_{\text {eff } 1}=25000 \mathrm{~K}, T_{\text {eff } 2}=5150 \mathrm{~K}$, with $R_{2} / R_{1}=$ 8.0. However it had been assumed that $n_{\mathrm{He} 1}=0.0$ and $\log g_{1}=5.0$. For this paper, the data were reanalyzed iteratively with the optical data (see below). Consequently, quite different model atmosphere grids were adopted in the final analysis, with $n_{\mathrm{He} 1}=0.95$ and $\log g_{1}=3.6$. Interstellar extinction was still found to be negligible, but the different distribution of opacity in the hydrogen-deficient atmosphere of the hot star resulted in a higher $T_{\text {eff }}$ being obtained for both components.

With $T_{\text {eff } 1}=28600 \pm 1000 \mathrm{~K}$, the earlier measurement of $\sim 31000 \mathrm{~K}$ (Ferguson et al. 1984) was approximately recovered. However, with $T_{\text {eff } 2}=5840 \pm 960 \mathrm{~K}$, the cool star appears to be somewhat hotter than the K3 spectral type indicated before. The broader spectral range covered by our data should lead to a more robust result than the flux ratio method (Wade 1980). It is noted that the errors are formal errors and do not allow for systematic errors as may be introduced, for example, by an inappropriate choice for unconstrained model atmosphere parameters. The relative radii of the two stars, given by their angular diameters, is $R_{2} / R_{1}=7.44 \pm 0.03$. Other parameters of the fit are given in Table 3 . The best-fit model flux distribution is shown together with the data in Fig. 2.

\subsection{Spectrum synthesis}

Much more information is available in the line spectrum than can be obtained from photometry alone. The object of spectrum synthesis is to find, given assumptions that have been introduced already, a model for the overall spectrum that best matches the observation by, for example, minimizing the square of residuals between model and data. Techniques for doing this with single stars have been described already (Jeffery et al. 2001). The extension to binary stars has been developed by Aznar Cuadrado \& Jeffery (in preparation) and described by Aznar Cuadrado (Aznar Cuadrado 2001).

Under perfect conditions (e.g. noise-free data), a residual-minimization procedure could solve for many free parameters simultaneously. In practice, it is necessary to hold most parameters fixed while solving for two or three at a time, and iterating around several parameters until an optimum solution is obtained. With the assumptions introduced already, the final solution for BI Lyn is given in Table 3. The errors cited in fitted quantities are formal errors from the $\chi^{2}$ minimization. Other errors have been propagated from these. Systematic errors (e.g. choice of $\left.\left[\beta_{\mathrm{Fe}}\right], v_{\mathrm{t}}\right)$ can significantly affect these.

A free spectroscopic solution for effective temperature gave $T_{\text {eff } 1}=30.1 \mathrm{kK}$, but could not provide $T_{\text {eff } 2}$ because of a lack of temperature-sensitive diagnostics. To maintain consistency in the radius ratio, the spectrophotometric solutions for $T_{\text {eff } 1}$ and $T_{\text {eff } 2}$ were retained.

The surface gravity and helium abundance of the hot star were well determined spectroscopically; there is negligible rotational broadening (at the instrumental resolution) in the line profiles. It is clear that the hot component is a hydrogen-deficient giant with $\log g_{1}=3.6 \pm 0.1$ with an atmosphere containing some 5 per cent hydrogen and 95 per cent helium (by number).

The cool star is more difficult to analyse, mainly because of substantial rotation broadening $v \sin i_{2}=$ $120 \pm 20 \mathrm{~km} \mathrm{~s}^{-1}$. Metallicity, microturbulent velocity, helium abundance and gravity all affect the strength of the metal line spectrum, which is then heavily smeared by the rotation. The infrared calcium triplet could provide 

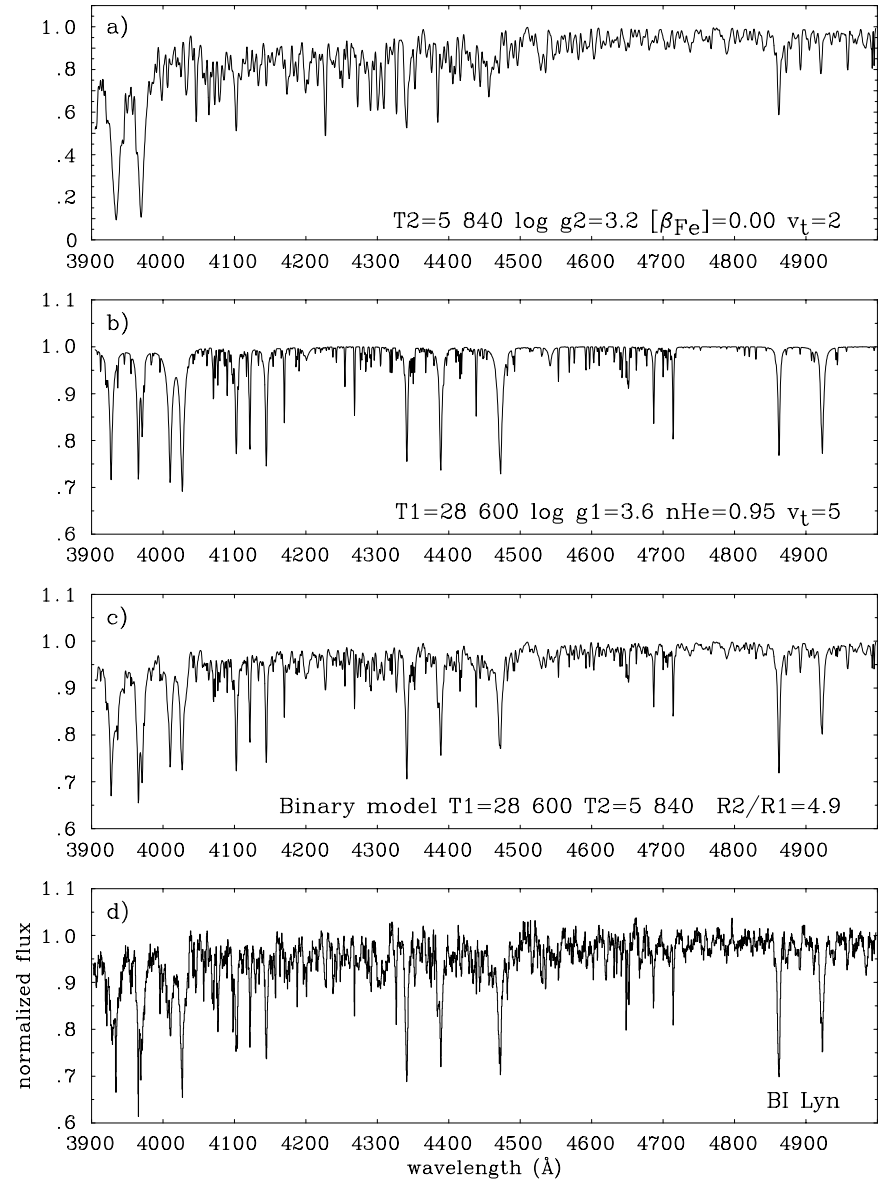

Fig. 3. Normalized blue spectrum of BI Lyn (bottom: d)) together with a best fit composite model spectrum c) formed by adding models with a) $T_{\text {eff } 2}=5840 \mathrm{~K}, \log g_{2}=3.2$, $\left[\beta_{\mathrm{Fe}}\right]_{2}=0.00$ (top) and b) $T_{\text {eff } 1}=28600 \mathrm{~K}, \log g_{1}=3.6$, $n_{\mathrm{He} 1}=0.95$ assuming that the relative radii $R_{2} / R_{1}=4.9$. The model spectra have been velocity shifted and degraded to match the observed spectral resolution (1 A) (WHT images: $337312+337313+337318+337319+337416+337418+$ 337419). A detailed comparison between the observed spectrum and best-fit model is shown in Fig. 4.

a good gravity indicator if $\left[\beta_{\mathrm{Fe}}\right]$ were known. Assuming $\left[\beta_{\mathrm{Fe}}\right]_{2}=0.0$ we find $\log g_{2}=3.2 \pm 0.3$.

With the assumptions given and parameters deduced, a free solution gives $R_{2} / R_{1}=4.9 \pm 0.5$ in both blue and infared spectral regions. This is determined solely by the strength of the cool star absorption spectrum, so is clearly assumption dependent. Given the nature of these, it is satisfactory that $R_{2} / R_{1}$ is within $40 \%$ of the value obtained from photometry.

The best-fit model spectra for both hot and cool stars, their sum according to the given relative radii and a comparison with the observed spectrum are given for all spectra regions in Figs. 3-6.

\subsection{Stellar dimensions}

Given the measured dimensions of BI Lyn, a number of other properties including masses, luminosities and
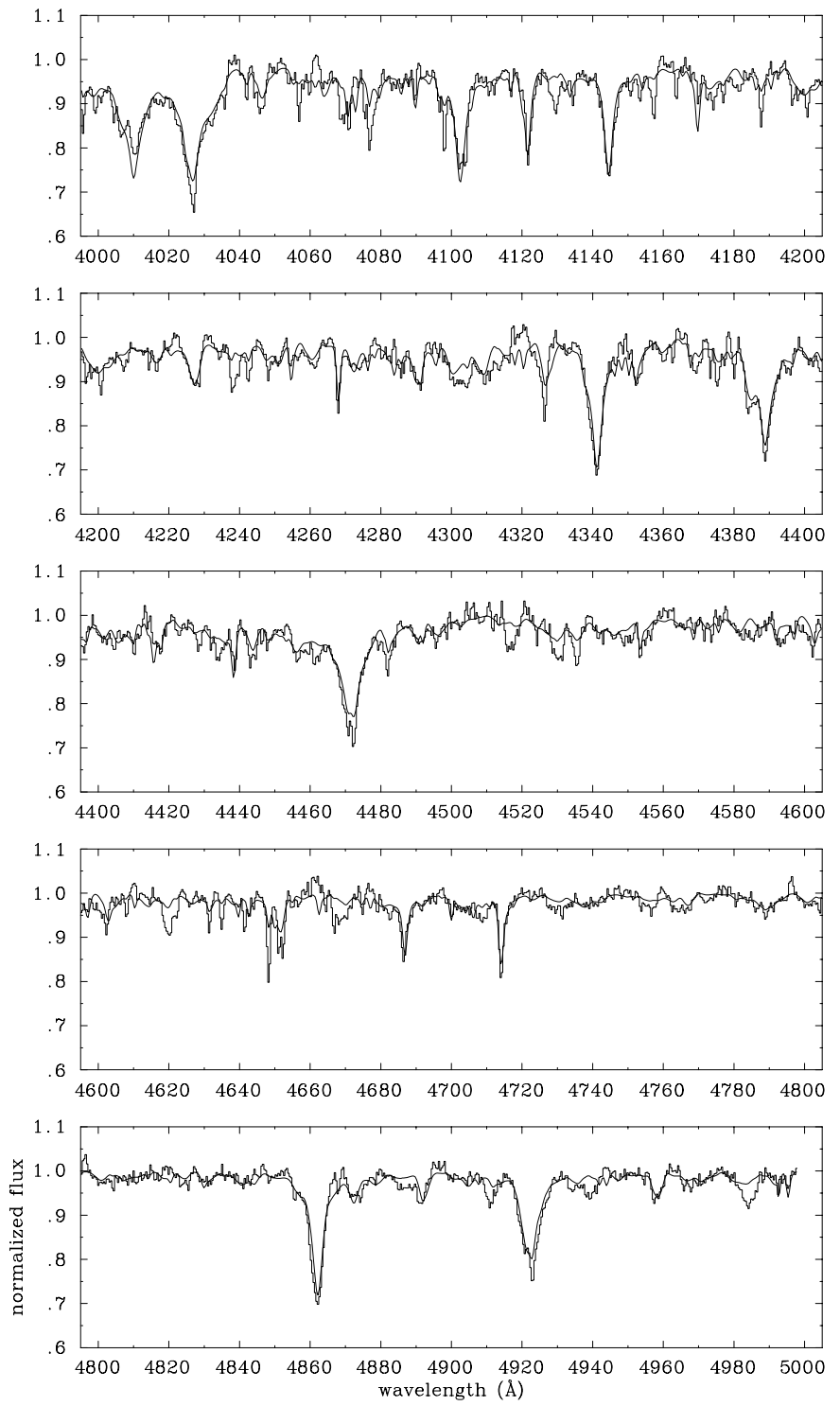

Fig. 4. Normalized blue spectrum of BI Lyn (histogram) together with the best fit composite model spectrum (polyline: see Fig. 3c) showing the fit in detail.

distance may be estimated. However these depend on which value for $R_{2} / R_{1}$ is adopted, an estimate for the hot star mass $M_{1}$ and the quality of the $\log g_{2}$ measurement. Possible values, given choices for each of these parameters, are shown in Table 4. The given distance is derived from the measured angular diameter and the derived radius of the cool star $\left(d=R_{2} / \theta_{2}\right)$. A similar result is obtained from the apparent visual magnitude and derived luminosities.

These solutions must be reconciled with the position of BI Lyn which, with $l^{\mathrm{II}}=182^{\circ}, b^{\mathrm{II}}=+42^{\circ}$, is substantially $(\gtrsim 3 \mathrm{kpc})$ out of the Galactic plane in the anticentre direction. It therefore seems preferable to seek the lowest possible mass for the cool star in which case a solution with $M_{1} \sim 0.5 M_{\odot}$ and $M_{2} \sim 5 M_{\odot}$ is indicated. The latter is comparable with the companion star mass estimated for another hydrogen-deficient binary $-v$ Sgr (Schönberner \& Drilling 1983; Dudley \& Jeffery 1990). 

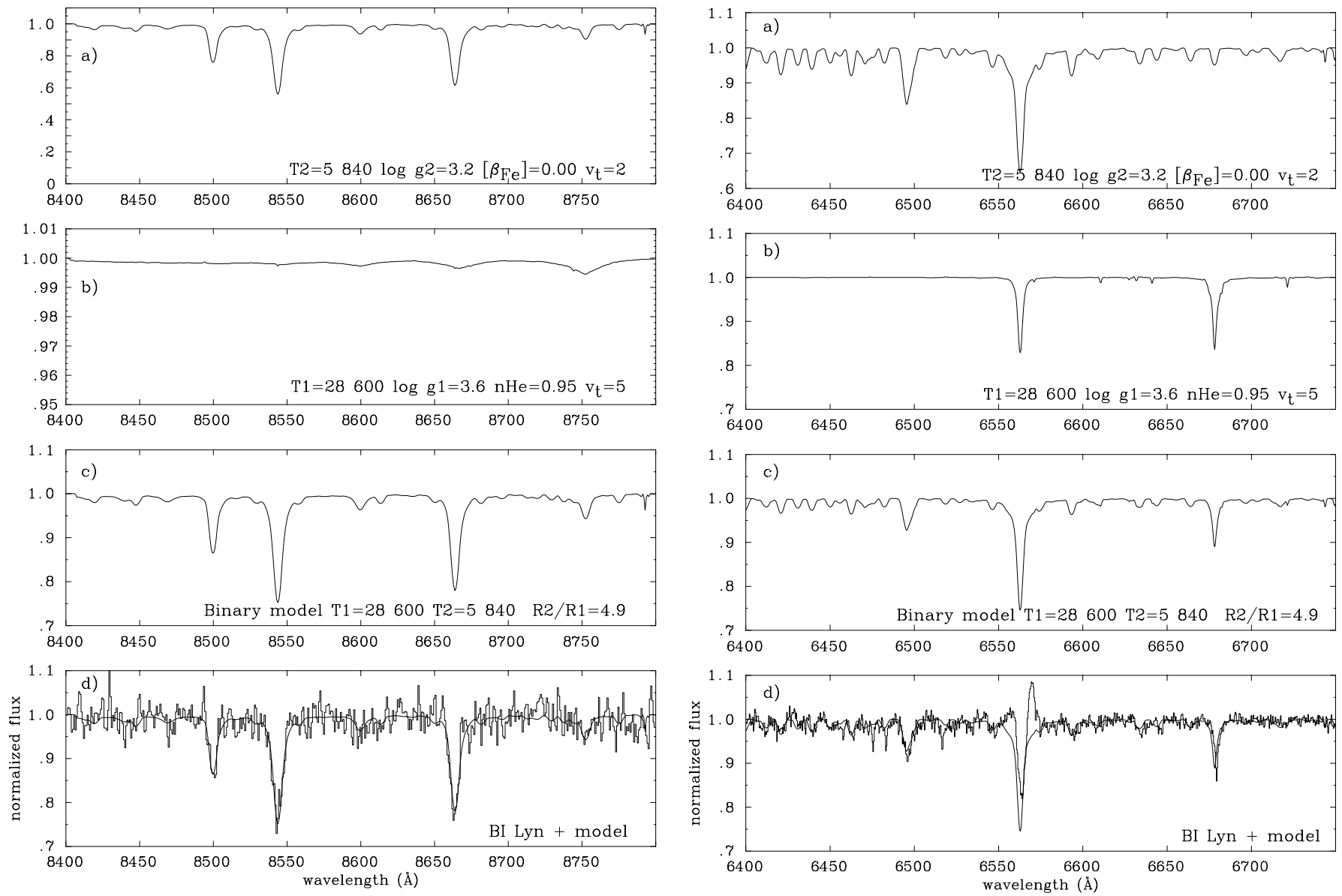

Fig. 5. As Fig. 3 in the region of the infared calcium triplet (INT image: 155728).

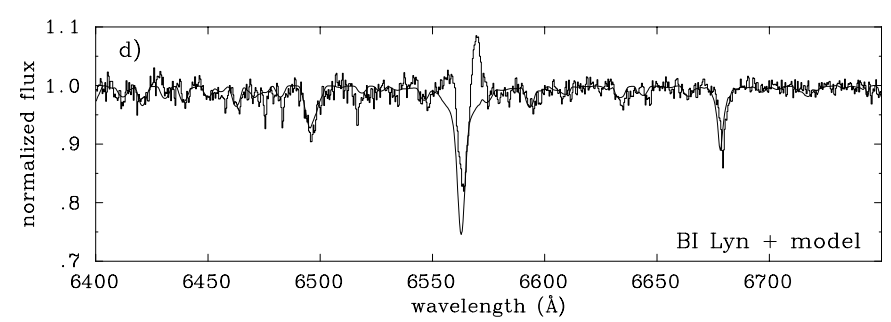

Fig. 6. As Fig. 3 in the region of $\mathrm{H} \alpha$ (WHT images: $337414+$ $337415+337417)$.

The referee has rightly pointed out that the detailed

A better solution might be achieved if we have overestimated $g_{2}$ by, for example, placing the continuum around the infrared calcium triplet too low or underestimating the hot star flux at these wavelengths. With $M_{1} \sim 0.5 M_{\odot}$ and $\log g_{2} \sim 2.9$ or 2.5 , a cool star mass $M_{2} \sim 2.4$ or $1 M_{\odot}$ would be easier to reconcile with the Galactic position.

In any event, the components must be highly evolved. A solution comprising a $0.5 M_{\odot}$ B-type helium star and a 1-5 $M_{\odot}$ G-type giant (luminosity class II - III) is consistent with the observational data presented in this paper. It is suggested that BI Lyn is a post common-envelope binary in which the primary (helium star) has almost completely shed its outer hydrogen-rich envelope together with substantial angular momentum. A large fraction of this has been transferred to the cooler star. The envelope of the cool star may not yet be in thermal equilibrium - there may still be a main-sequence star underneath giving it the semblance of a giant. This would account for the apparently advanced evolutionary state of the cool star at the same time as the hot star is in a relatively short-lived phase of evolution.

The surface gravity for the hot star places it on an evolutionary track for a post-AGB star of $\sim 0.5 M_{\odot}$ (Schönberner 1983). results of our analysis are subject to the LTE assumption used in the analysis of the early-type star. Being a giant, departures from LTE can be significant and will affect, in particular, the predicted equivalent widths of the He I lines. Other spectral features may also be affected. NonLTE calculations for hydrogen-poor hydrogen-helium atmospheres have been available for some years, but fully line-blanketed NLTE models for hydrogen-poor atmospheres with $T_{\text {eff }} \sim 30000 \mathrm{~K}$ have not yet been successfully computed (Rauch 1996). Their eventual arrival will affect the detailed results presented here but not the overall conclusions concerning the dimensions of BI Lyn.

\section{Variability}

\subsection{Photometry}

BI Lyn has been found to be variable in light with an amplitude of $\sim 0.1 \mathrm{mag}$ and a period of $0.33818 \mathrm{~d}$ (Lipunova \& Shugarov 1990, 1991). Reports of other periodicities (280 s: Lipunova \& Shugarov 1991, 1117 s: Kuczawska et al. 1993) have not been confirmed. These find possible explanations in a CV model as the orbital period and oscillations of the white dwarf respectively. An orbital period of $0.34 \mathrm{~d}$ was not detected spectroscopically 


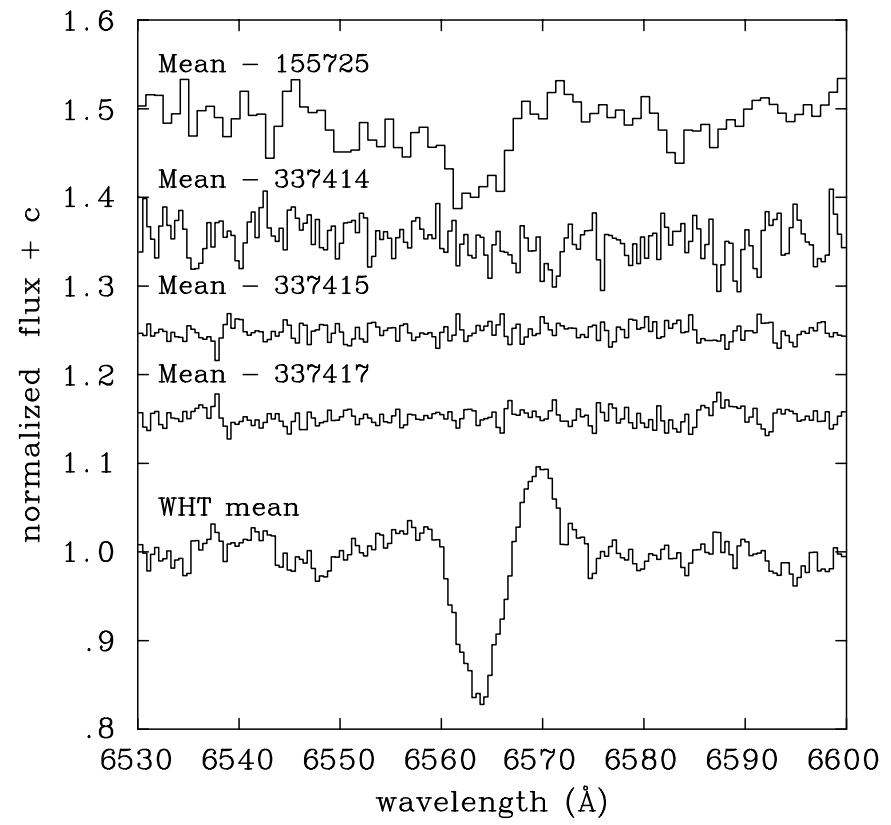

Fig. 7. Residuals of four spectra with respect to the mean WHT spectrum in the region of $\mathrm{H} \alpha$, labelled by image number. Only the INT spectrum shows a significant change in the absorption strength.

(Orosz et al. 1997). The $0.34 \mathrm{~d}$ period cannot be associated with the cool star rotation; a star with $R=9 R_{\odot}$, $T_{\text {eff }}=5840 \mathrm{~K}$ and $\log g=3.2$ has a minimum rotation period of $2.6 \mathrm{~d}$.

A period of $\sim 0.3 \mathrm{~d}$ can be explained by pulsations (radial or non-radial) in a luminous hot helium star. A corollary would be V2076 Oph (Lynas-Gray et al. 1987), a luminous helium star with $T_{\text {eff }} \sim 32000 \mathrm{~K}$, $\log g \sim 3$ and non-radial pulsation periods $\sim 0.7$ and 1.1 days. The observed periods of these strange-mode pulsations (Saio 1995) depend critically on the stellar mass, luminosity and which modes are most excited. V2076 Oph is thought to have a mass $\sim 0.7 M_{\odot}$ (Lynas-Gray et al. 1987).

\subsection{Radial velocity}

Given the resolution and inhomogeneity of the spectra, the short time base over which observations were obtained and the rotational broadening of the cool star spectrum, no significant changes in the radial velocity of either component could be identified (Table 2). The mean difference in velocity of the two components is negligible. High-resolution studies of a specific spectral region over an extended interval will be necessary to establish the orbital period and velocity amplitudes in this system. These will be extremely important observations and should be undertaken as a matter of urgency since they will give the mass ratio directly and independently from the spectroscopic analysis.

\section{3. $H \alpha$}

Variations in $\mathrm{H} \alpha$ are more difficult to interpret reliably, particularly since the promised report of 1995 March observations (Wade \& Potter 1995; Orosz et al. 1997) has still to appear. The available data describe a broad emission with a variable and narrower absorption component. We observed a change in the absorption strength of $\mathrm{H} \alpha$ between 1998 and 1999, but not during the short interval of the 1999 observations (Fig. 7). A rapid increase in absorption strength has been reported elsewhere (Wade \& Potter 1995). Such phenomena have previously been seen in hydrogen-deficient supergiant binaries (e.g. $v$ Sgr) on a timescale comparable with their orbital periods.

$v$ Sgr is a single-lined spectroscopic binary with an orbital period of 138 days (Wilson 1914). The primary is an early-type supergiant with $T_{\text {eff }} \sim 12000 \mathrm{~K}$ (Dudley \& Jeffery 1993) and an extremely low hydrogen surface abundance (Schönberner \& Drilling 1983). Being of the 6th magnitude, it has been scrutinized for over a century (Campbell 1899). It is interesting to review some of the reports concerning $\mathrm{H} \alpha$ :

- "Intensity variations were found in the emission and absorption of hydrogen lines" (Seydel 1929);

- "At $\mathrm{H} \alpha$ a very broad, weak emission band, roughly $8 \AA$ in width, can be seen" (Greenstein 1943);

- "One strong P Cygni outburst was visible in the hydrogen lines in the envelope of the system. A double strong, deep, absorption at $\mathrm{H} \alpha$ appeared..." (Greenstein 1950);

- "Stationary emission lines of [...] H $\alpha$ occur in the envelope of the system" (Greenstein 1950);

- "Many investigators have reported that the displaced $\mathrm{H} \alpha$ absorption line appears during an interval of about 40 days centered about the phase... at which the primary is farthest from us (e.g. Bidelman 1949; Hack 1960)" (Nariai 1967);

- "... the gas flow was not observed during cycles 43 to $98 \ldots$ It is suggested that the jet appears periodically..." (Nariai 1967);

More recent data (Frame et al. 1995) confirm the strongly variable nature of $\mathrm{H} \alpha$ absorption and emission. Absorption episodes are roughly correlated with orbital phase although they may switch off for several cycles at a time. The preferred model is that of a supersonic jet (Nariai 1967) generated by material passing through the inner Lagrangian point on to the secondary and which eclipses an extended envelope around the primary responsible for the broad emission. Similar phenomena have been seen in the three other known hydrogendeficient binaries KSPer (Nariai 1972), V426Car = CPD-58 2721 (Frame et al. 1995) and HDE320156 = LSS4300 (Frame et al. 1995).

Although the data are sparse, the qualitative behaviour of $\mathrm{H} \alpha$ in $\mathrm{BI}$ Lyn and $v$ Sgr may be similar. 
Consequently a similar interpretation involving a circumstellar envelope and a gas-stream flowing through the inner Lagrangian point may be appropriate. The rapid increase in absorption reported in $\mathrm{H} \alpha$ (Wade \& Potter 1995) would correspond to eclipse of the circumstellar material by the gas stream. Clearly, more detailed work including determination of the orbital period and the phase-dependency of the $\mathrm{H} \alpha$ profile will be required to confirm this hypothesis.

\section{Conclusion}

We have obtained intermediate dispersion spectra of the supposed hot subdwarf binary BI Lyn. These have been analysed, together with the overall flux distribution, to establish the dimensions of both hot and cool components. The hot component is clearly demonstrated to be a lowmass hydrogen-deficient star with low surface gravity. The cool component is most likely a giant of approximately one to a few solar masses. The hydrogen-deficiency of the hot star is probably the result of a common-envelope phase during which the outer envelope was entirely removed or transferred to the cool companion. The hot star luminosity suggests that it lies on a post-AGB evolution track. Previous reports of variability in light and $\mathrm{H} \alpha$ emission in BI Lyn are consistent with the behaviour of other $\mathrm{H}$-deficient giants, being caused by pulsations and mass transfer respectively. Further observations are required to determine the orbital period and mass ratio, to verify the pulsation hypothesis and to correlate the $\mathrm{H} \alpha$ behaviour with orbital phase.

Note added in proof: A striking similarity between BI Lyn and HD 128220 has been drawn to our attention. The latter is a binary containing an $\mathrm{O}$ subdwarf and a rapidly rotating G-type primary, probably spun up by mass exchange (Howard I. D., \& Heber U. 1990, PASP, 102, 912). Thus both systems consist of a hot post-AGB star and a more-massive rapidly-rotating cool companion.

Acknowledgements. This research has made extensive use of software provided through UK PPARC Collaborative Computational Project No. 7 for "the Analysis of Astronomical Spectra", software and facilities provided through the UK PPARC Starlink project and the SIMBAD database, operated at CDS, Strasbourg, France. We acknowledge financial support from the Northern Ireland Department of Culture, Arts and Leisure.

\section{References}

Aznar Cuadrado, R. 2001, Ph.D. Thesis, Queens University Belfast, submitted

Aznar Cuadrado, R., \& Jeffery, C. S. 2001, A\&A, 368, 994

Bidelman, W. P. 1949, ApJ, 109, 544

Campbell, W. W. 1899, ApJ, 10, 241

Downes, R., Webbink, R. F., \& Shara, M. M. 1997, PASP, 109, 345

Dudley, R. E., \& Jeffery, C. S. 1990, MNRAS, 247, 400

Dudley, R. E., \& Jeffery, C. S. 1993, MNRAS, 262, 945

Ferguson, D. H., Green, R. F., \& Liebert, J. 1984, ApJ, 287, 320

Frame, D. J., et al. 1995, MNRAS, 276, 383

Greenstein, J. L. 1943, ApJ, 97252

Greenstein, J. L. 1950, AJ, 55, 72

Hack, M. 1960, Contr. Milano Merate Obs., No. 215

Jeffery, C. S. 1991, CCP7 Newslett., No. 16, 16

Jeffery, C. S., \& Pollacco, D. 1998, MNRAS, 298, 179

Jeffery, C. S., Drilling, J. S., \& Heber, U. 1987, MNRAS, 226, 317

Jeffery, C. S., Lester, J., \& Short, I. 1996, CCP7 Newslett., No. 24,13

Jeffery, C. S., Woolf, V. M., \& Pollacco, D. L. 2001, A\&A, 376, 497

Kazarovets, E. V., Samus, N. N., \& Goranskij, V. P. 1993, IBVS, 3840

Kuczawska, E., Mikolajewski, M., \& Kirejczyk, K. 1993, IBVS, 3845

Kurucz, R. L. 1979, ApJS, 40, 1

Kurucz, R. L. 1993, SAO KURUCZ CD-ROM, No. 18

Kurucz, R. L. 1991, in Stellar Atmospheres: Beyond Classical Models, ed. L. Crivellari, I. Hubeny, \& D. G. Hummer, NATO ASI Ser., vol. C341 (Kluwer, Dordrecht), 441

Lipunova, N. A., \& Shugarov, S. Yu. 1990, JAVSO, 19, 40

Lipunova, N. A., \& Shugarov, S. Yu. 1991, IBVS, 3580

Liu, W., Hu, J. Y. 2000, ApJS, 128, 387

Lynas-Gray, A. E., Kilkenny, D., Skillen, I., \& Jeffery, C. S. 1987, MNRAS, 227, 1073

Nariai, K. 1967, PASJ, 19, 564

Nariai, K. 1972, PASJ, 24, 495

Orosz, J. A., Wade, R. A., \& Harlow, J. J. B. 1997, AJ, 114, 317

Rauch, T. 1996, Hydrogen Deficient Stars, ed. C. S. Jeffery, \& U. Heber, ASP Conf. Ser., 96, 174

Ritter, H., \& Kolb, U. 1998, A\&AS, 129, 83

Saio, H. 1995, MNRAS, 277, 1393

Schönberner, D. 1983, ApJ, 272, 708

Schönberner, D., \& Drilling, J. S. 1983, ApJ, 268, 225

Seydel, F. L. 1929, Publ. Amer. Astr. Soc., 6, 278

Wade, R. A. 1980, Ph.D. Thesis, California Institute of Technology

Wade, R. A., \& Potter, D. 1995, BAAS 27Q.877

Walker, H. J., \& Schönberner, D. 1981, A\&A, 97, 291

Wilson, R. E. 1914, Lick Obs. Bull., 8, 132 